This item was submitted to Loughborough's Research Repository by the author.

Items in Figshare are protected by copyright, with all rights reserved, unless otherwise indicated.

\title{
H2 LMI-based robust control for damping oscillations in power systems
}

PLEASE CITE THE PUBLISHED VERSION

PUBLISHER

(C) IEEE

VERSION

VoR (Version of Record)

LICENCE

CC BY-NC-ND 4.0

REPOSITORY RECORD

Zolotas, Argyrios C., Petr Korba, B. Chaudhuri, and I.M. Jaimoukha. 2019. "H2 Lmi-based Robust Control for Damping Oscillations in Power Systems". figshare. https://hdl.handle.net/2134/4316. 
This item was submitted to Loughborough's Institutional Repository (https://dspace.lboro.ac.uk/) by the author and is made available under the following Creative Commons Licence conditions.

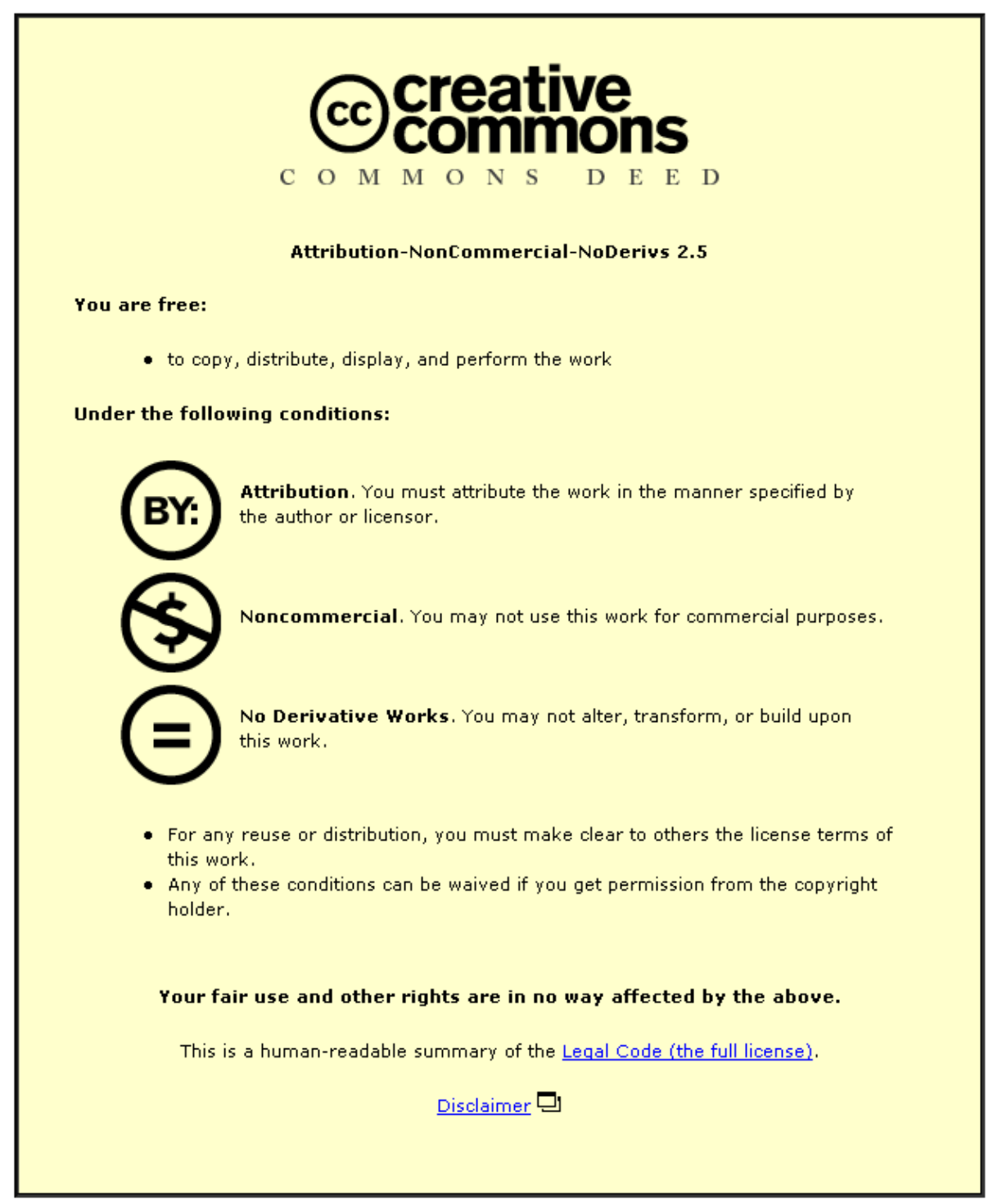

For the full text of this licence, please go to: http://creativecommons.org/licenses/by-nc-nd/2.5/ 


\section{$\mathscr{H}_{2}$ LMI-based Robust Control for Damping Oscillations in Power Systems}

\author{
Argyrios C. Zolotas \\ Department of EEE \\ Loughborough University \\ Loughborough LE11 3TU, UK \\ a.zolotasieee.org
}

\author{
Petr Korba \\ ABB Switzerland Ltd. \\ Corporate Research \\ Baden-Dättwil, Switzerland \\ petr.korbadch.abb.com
}

\author{
Balarko Chaudhuri, Imad M. Jaimoukha \\ Department of EEE, Imperial College \\ London SW7 2AZ, UK \\ b. chaudhuridimperial.ac.uk \\ i.jaimouka@imperial.ac.uk
}

\begin{abstract}
This paper presents a study on a robust $\mathscr{H}_{2}$ LMI-based damping control scheme for improving the interarea mode oscillations of power systems. A reduced order centralised controller is designed for a 16-machine, 5area power system (138th order) model reinforced with a Thyristor Controlled Series Capacitor (TCSC) to improve the damping of the critical inter-area modes by employing appropriate signal measurements. The effectiveness of the Linear Matrix Inequalities (LMI) formulation, using pole location constraints, of the problem is illustrated. Robustness to faults and unknown disturbances is guaranteed via the design procedure. The performance of the designed system is assessed in the frequency domain and via appropriate timedomain simulations based upon the non-linear model under various scenaria.
\end{abstract}

Keywords: Power systems, inter-area oscillations, Linear Matrix Inequalities, $\mathscr{H}_{2}$ control, robust control.

\section{Introduction}

One of the principal concerns for the electric power system operators is damping inter-area oscillations [1]. The problem has become even more challenging, owing to the ever increasing power exchange between utilities over the existing transmission network [2]. Thus, secure operation of power systems necessitates the application of robust controllers to appropriately damp these inter-area oscillations [3]. The most commonly used device for such a purpose is a Power System Stabilizer (PSSs), see e.g. [4], [5]. However, Flexible AC Transmission Systems devices - widely known as FACTS - are receiving growing attention nowadays as alternatives to transmission system reinforcement which is otherwise restricted due to economic and environmental considerations. Besides power flow and voltage control, supplementary control can be incorporated to these FACTS devices to damp inter-area oscillations at relatively small additional cost [6], [7], [8]. The objective of the presented control design exercise is to guarantee adequate damping under plausible operating conditions via the use of a Thyristor Controlled Series Capacitor (TCSC). It is worth noting that the complex nature of the interactions in the inter-area modes of the system, make the control design task rather challenging.
Many researchers have investigated $\mathscr{H}_{\infty}$ control methods in power systems [9], [10], [11], [12], [13], acknowledging the fact that care should be taken in selecting weighting functions for a proper design procedure. LQG control approaches using different FACTS devices have been presented for closed loop identification in [15], [14], and PSSs for small systems in [16]. Another study, [17], looked at an LQG/LTR design with a TCSC device. However, it has been applied to a rather small SISO system addressing the LTR problem from a simulation point of view.

In this paper, a robust $\mathscr{H}_{2} / \mathrm{LQG}$ controller is designed within the Linear Matrix Inequalities (LMI) framework. In fact LQG is regarded as special case of a $\mathscr{H}_{2}$ controller. However, we make use of the additional capability to incorporate multiple objectives in the problem configuration (thanks to the LMI reformulation of the entire controller design), e.g. pole-placement constraints and additional upper bounds on infinity norms of the closed loop functions. In addition to robust stabilisation of the considered power system, a minimum damping ratio is also ensured when using the LMI-based design. The system consists of a 16machines, 5-area power network controlled by a designed (centralised) TCSC device using three remote (global) measurement signals. Extensive emphasis on the model reduction simplifies the system structure of the $138^{\text {th }}$ order equivalent model representation of a large power network for a more appropriate model-based control design, while the system performance objective is tackled via the $\mathscr{H}_{2}$ minimisation index. The effectiveness of the design is illustrated via both frequency domain assessment and non-linear time domain simulations for various operating conditions. The study illustrates the fact that multiple swing modes can be damped through a single actuator (FACTS) using appropriate control design methodology in an advanced multi-variable control framework.

This paper is organized as follows: Section 2 briefly describes the basic notation used in the paper. The power system model under consideration is depicted in Section 3. Section 4 discusses on the model reduction approach. Preliminaries on control design as presented in Section 5, while the control design procedure for the case study is discussed in Section 6. The obtained results are presented in Section 7. Finally, conclusions are drawn in Section 8. 


\section{Notation}

The notation is fairly standard. The transfer matrix in terms of state-space data, for a continuous time, Linear Time Invariant (LTI) state space system, is given in a packed form by $G(s) \stackrel{s}{=}\left[\begin{array}{l|l}A & B \\ \hline C & D\end{array}\right]$ denoting $G(s)=D+C(s I-A)^{-1} B$. We will make clear in the paper whether the system is proper or strictly proper. Moreover, the study utilises the generalised regulator configuration for standardising feedback arrangements [18] and is briefly described here (refer to Fig.1). Let $P(s)$ define a transfer function matrix with the following state-space representation

$$
P(s) \stackrel{s}{=}\left[\begin{array}{l||l|l}
A & B_{1} & B_{2} \\
\hline \hline C_{1} & D_{11} & D_{12} \\
\hline C_{2} & D_{21} & D_{22}
\end{array}\right]=\left[\begin{array}{l|l}
P_{11} & P_{12} \\
\hline P_{21} & P_{22}
\end{array}\right]
$$

where $P_{i j}(s)=C_{i}(s I-A)^{-1} B_{j}+D_{i j}$

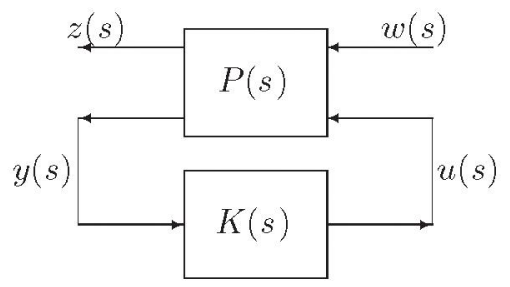

Fig. 1. The Generalised Regulator Configuration

for $\left|I-P_{22} K\right| \neq 0 . P(s)$ is the "generalised plant" (nominal plant and weights if any), $u$ the control variables, $w$ the exogenous inputs (i.e. disturbances, commands, noises), $y$ the measured variables and $z$ the regulated outputs. In fact $z(s)=\mathscr{F}_{L}(P, K) w(s)$, where

$$
\mathscr{F}_{L}(P, K) \triangleq P_{11}+P_{12} K\left(I-P_{22} K\right)^{-1} P_{21}
$$

\section{System Modeling and Design Require- ments}

\subsection{Study System}

The damping control design under study consists of a 16machine, 5-area power system and is depicted in Fig. 2. The diagram represents an approximate model of the New England (NETS) and New York (NYPS) interconnected network. More details on the system description and its characteristics including machine, excitation system and network parameters can be found in [11]. To facilitate the required power transfer, a TCSC device is installed in the line between buses \#18 and \#50. The small signal dynamic model of the TCSC is shown in Fig. 3 (more details on this can be found in [23]).

Table I lists the results of the eigen-analysis verifying the presence of four inter-area modes from which the first three are poorly damped requiring damping control action (details can be found in [11]). Note that the fourth inter-area mode is not significant as it settles within the specified limit of $12-15 \mathrm{~s}$ (the damping ratio is as poor as the other three modes, but the higher frequency of oscillation is resulting in an early settling in time). The TCSC controller (centralised) needs to provide supplementary damping control action to all critical inter-area modes of interest. The most effective measurements for control were found to be the power-flow signals in the lines between buses \#51- \#45 (P51;45), \#18\#16 (P18;16) and \#13-\#17 (P13;17) respectively [23], based on the results of the modal analysis. These are the lines which are carrying large amounts of power from Area 3, Area 4 and the equivalent generation G13 respectively.

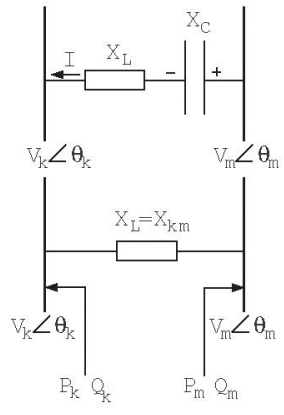

Power Injection Model

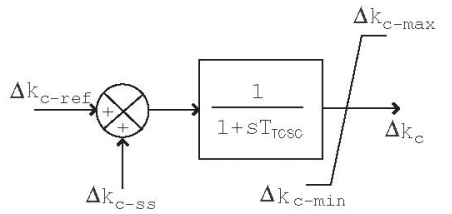

Small-signal dynamic model
Fig. 3. Thyristor Controlled Series Capacitor (TCSC) diagram

\subsection{Damping Control Design Objectives}

The designed controller should provide a minimum level of damping in the steady-state condition after a major disturbance in all the key interconnections. The aforementioned minimum level of damping corresponds to settling of interarea oscillations within $10-15$ seconds [19]. Power systems operate over a wide range of operating conditions and there is usually uncertainty associated with the simulation models employed for evaluating the performance. Under such circumstances the controller should be robust and subject to having minimal sensitivity to various system operating conditions and component parameters. Furthermore, interactions with high frequency phenomena, turbine-generator torsional vibration and other resonances in the $\mathrm{AC}$ transmission network should be kept minimal.

\section{Model Reduction}

Modern control design processes such as LQG, $\mathscr{H}_{2}, \mathscr{H}_{\infty}$, (both via analytical and LMI-based solutions) yield controllers of order at least equal to the order of the plant. Actually the controller order can be higher due to extra weighting functions merged in the design procedure. Model order reduction seeks a good low-order approximation of the original model to simplify the design procedure and thus the complexity of the designed controller. Hence, the central problem addressed is: Given a high-order linear model $G(s)$, 


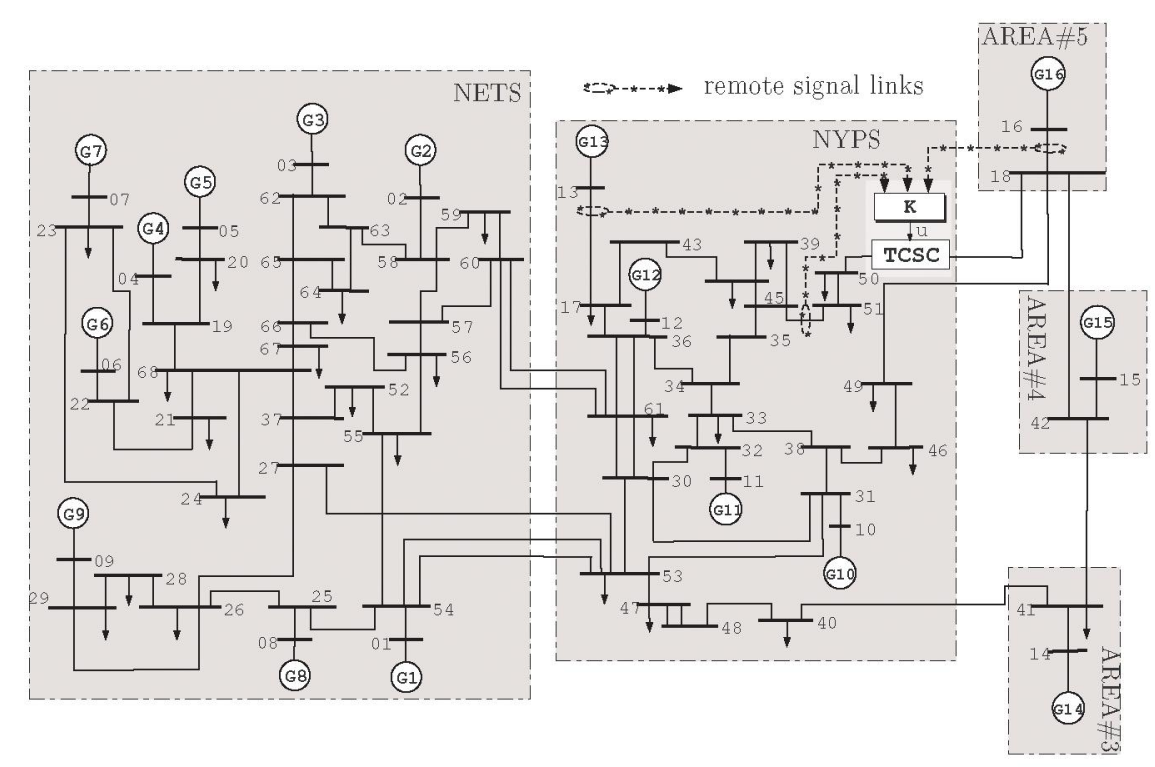

Fig. 2. 16-machine/5-area case study power system with a TCSC (FACTS) device

derive a low order approximation $G_{r}(s)$ such that the infinity norm of their difference $\left\|G-G_{r}\right\|_{\infty}$ is sufficiently small. The same applies in the controller reduction approach.

Our designs make particular use of the Schur Balanced Model Reduction method for both model and controller simplification [25], i.e. Compute the $k^{\text {th }}$ order reduced model $G_{r}(s)=C_{r}\left(s I-A_{r}\right)^{-1} B_{r}+D_{r}$ from an $n^{\text {th }}$ order full model $G(s)=C(s I-A)^{-1} B+D$ such that

$$
\left\|G-G_{r}\right\|_{\infty} \leq 2 \sum_{i=k+1}^{n} \sigma_{i}
$$

where $\sigma_{i}$ denotes the Hankel singular values of $G(j \omega)$, i.e. the square roots of the eigenvalues of their controllability and observability grammians $\sigma_{i}:=\sqrt{\lambda_{i}(P Q)}$, where $\lambda_{i}(P Q)$ is the $i$-th largest eigenvalue of $P Q$ and $P$ (controllability grammian), $Q$ (observability grammian) are the solutions of the following Lyapunov equations

$$
P A^{T}+A P+B B^{T}=0, \quad Q A+A^{T} Q+C^{T} C=0
$$

In cases where a large number of state variables is involved (i.e., $>1000$ ) one might employ numerical techniques, e.g. Krylov subspace based technique [20], as the analytical techniques alone will not work.

\subsection{Few remarks on model reduction for the power system study}

A realistic representation of the power system with supplementary damping controllers also includes appropriate washout and signal transmission delay blocks. In the context of this study, these blocks are chosen as:

$$
\begin{aligned}
& V_{1}(s)=\frac{10 s}{10 s+1}(\text { wash-out }) \\
& V_{2}(s)=\frac{1}{1+0.1 s}(\text { signal transmission delay })
\end{aligned}
$$

The time constants of the above filters can be adjusted by the designer in accordance with the actual disturbance caused typically by the adverse modes and measurement noise. However, performing model reduction on the plant incorporating these blocks proves insufficient in terms of proper approximation over all frequency ranges. In the context of this work we first reduce the order of the plant $G_{O}$ without the washout $\left(V_{1}\right)$ and delays $\left(V_{2}\right)$, and then merge the blocks $V_{2}, V_{1}$ into the reduced order equivalent $\hat{G}_{o}$. Note that the order of the reduced model for the design purposes - i.e. $V_{1} V_{2} \hat{G}_{o}$ - will be slightly increased due to the extra blocks, albeit the controller design will have essential information for the washout and signal delay characteristics. Note that identical washout and transmission delay blocks were assumed for each output channel, thus without loss of generality these were replaced by a single washout and a single delay block at the input channel for the design process.

A $9^{\text {th }}$ order reduced model $G_{r}$ (including 7 states from the reduced model and 2 additional states from the filters) was found appropriate for control design, as it includes sufficient information for the main modes of interest and system stability. The principal gains (singular value plot) of reduced and full order plants is shown in Fig. 4, with the reduced plant being a very good approximation especially within the frequency range of interest (by definition the largest singular of the three-output/one-input system transfer function is depicted in the plot).

\section{Preliminaries}

\section{$5.1 \mathscr{H}_{2}$ Control}

The $\mathscr{H}_{2}$ norm of $G(s)$ is given by

$$
\|G(s)\|_{2} \triangleq \sqrt{\frac{1}{2 \pi} \int_{-\infty}^{\infty} \operatorname{tr}\left(G^{*}(j \omega) G(j \omega)\right) d \omega}
$$




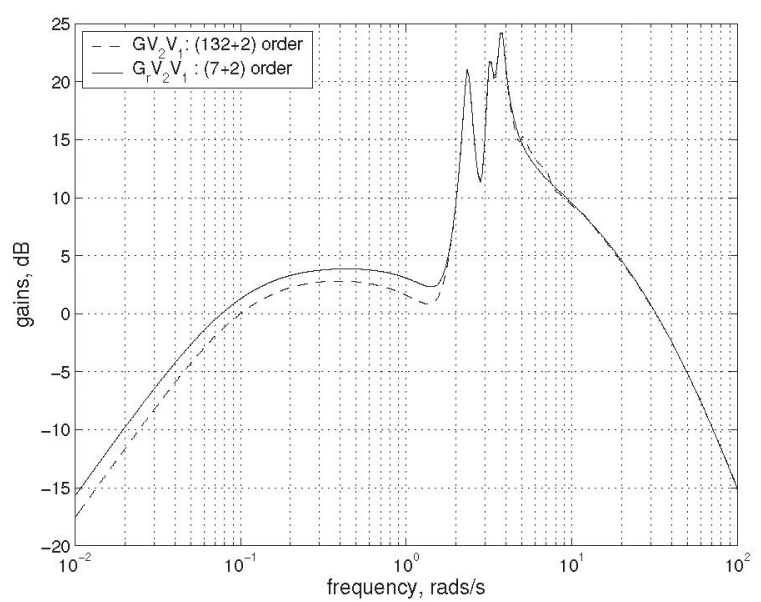

Fig. 4. Model reduction results for the plant

and it is finite only when $G(s)$ is strictly proper (which is the case for the model considered in this paper). Note that $\operatorname{tr}(A)$ denotes the trace of a square matrix $A$. $\mathscr{H}_{2}$ optimal control is concerned with finding a stabilising controller $K$ which minimizes

$$
\|F(s)\|_{2}=\sqrt{\frac{1}{2 \pi} \int_{-\infty}^{\infty} \operatorname{tr}\left(F^{*}(j \omega) F(j \omega)\right) d \omega}
$$

where $F \triangleq \mathscr{F}_{L}(P, K)$ in the generalised plant configuration. Also, the expected power in the regulated (error) signal $z$ is given by

$$
E\left\{\lim _{T \rightarrow \infty} \frac{1}{2 T} \int_{-T}^{T} z(t)^{T} z(t) d t\right\}=\|F\|_{2}^{2}\left(=\left\|\mathscr{F}_{L}(P, K)\right\|_{2}^{2}\right)
$$

Thus, by minimising the $\mathscr{H}_{2}$ norm, we minimize the RMS value of the regulated variable $z$, see Fig.1, [26].

\subsection{Linear Matrix Inequalities (LMI)}

LMIs arise in many problems from robust linear control theory, stochastic control, identification, etc. The problem is represented in a set of Linear Matrix Inequalities and then a convex optimisation approach is followed to give the solution. This technique has very attractive computational properties and in fact, its wide applicability justifies the increasing interest for LMI-based designs in the systems and control community [21]. The three generic LMI problems are

- the feasibility problem:

$$
\text { Find } x \in \mathbb{R}^{p} \text { such that } \mathscr{L}(x)<0
$$

- the linear program under LMI constraints:

$$
\text { Minimize } c^{T}(x) \text { subject to } \mathscr{L}(x)<0
$$

- the generalized eigenvalue minimization problem under LMI constraints:

$$
\text { Minimize } \lambda \text { subject to }\left\{\begin{array}{l}
\mathscr{C}(x)>0 \\
\mathscr{B}(x)>0 \\
\mathscr{A}(x)<\lambda \mathscr{B}(x)
\end{array}\right.
$$

The negative definite symmetric matrix $\mathscr{L}($.$) depends$ affinely on $x \in \mathbb{R}^{p}$ (decision variables). Possibilities include $\mathscr{H}_{2}$ or $\mathscr{H}_{\infty}$ designs, multi-objective $\mathscr{H}_{2} / \mathscr{H}_{\infty}$, gain scheduling, etc., assisted by useful software routines, i.e. see [28]. A particular feature of LMIs is the incorporation of pole placement objectives, This is formulated in terms of convex subsets (LMI regions) $\mathscr{R}$, of the complex plane

$$
\mathscr{D}=\left\{z \in C: L+M z+M^{T} \bar{z}<0\right\}
$$

where $M$ and $L=L^{T}$ are fixed real matrices. The matrixvalued function $f_{\mathscr{D}}(z)=L+M z+M^{T} \bar{z}$, is referred to as the characteristic function of $\mathscr{D}$. A general class of LMI regions exist for the above purpose, i.e. disks, conic sectors, vertical/horizontal strips, etc. or even intersections of the above. We will particularly concentrate on the 'conic sector', of inner angle $\theta$ and apex $x_{0}=0$, which defines a minimum damping for the dominant closed-loop poles, see Fig. 5. Its characteristic function is given by

$$
f_{\mathscr{D}}^{\operatorname{conic}}(z)=\left[\begin{array}{rr}
\sin \frac{\theta}{2}(z+\bar{z}) & \cos \frac{\theta}{2}(\bar{z}-z) \\
\cos \frac{\theta}{2}(z-\bar{z}) & \sin \frac{\theta}{2}(z+\bar{z})
\end{array}\right]
$$

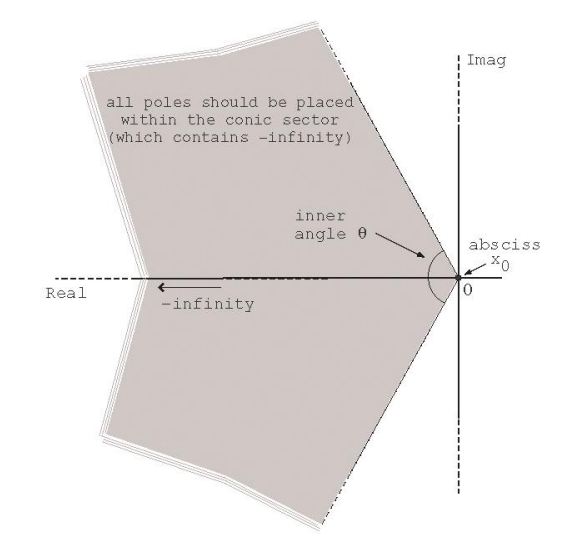

Fig. 5. Conic sector region for LMI pole placement

The damping ratio of the placed poles within the conic sector will be at least equal to $\cos \frac{\theta}{2}[28]$.

\section{Design Procedure}

This section presents two cases. Firstly the original LQG control problem formulated in the $\mathscr{H}_{2}$ framework is solved, and it is further enhanced via LMIs to include pole location constraints for the designed closed-loop. A brief discussion on further extensions of the design to include other objectives is also presented. The design procedure is based on the reduced order model, but the assessment is made using the full order model scenarios. Note that in both design cases the controller has 1 output and 3 inputs (i.e. is an $1 \times 3$ system, while the design model is a $3 \times 1$ system having 3 outputs and 1 input). 


\section{1 $\mathscr{H}_{2} /$ LQG control}

Linear Quadratic Gaussian control is well documented in the literature [27],[26]. Basically, two problems are solved: (i) find the optimal control using state feedback which minimizes the cost function

$$
J=\lim _{T \rightarrow \infty} \frac{1}{T} E\left\{\int_{0}^{T}\left[x^{T} Q x+u^{T} R u\right] d \tau\right\}
$$

and (ii) find the optimal state estimate $\hat{x}$ of $x$ to minimize $E\left\{[x-\hat{x}]^{T}[x-\hat{x}]\right\}$. The weighting matrices $Q$ (states weights, pos. semidefn.), $R$ (control weigh, pos. defn.) for the control problem, and $W$ (process noise covariance, pos. semidefn.), $V$ (measurement noise covariance, pos. defn.) for the estimation problem, are the tuning parameters. The dual procedure can be also followed, i.e. solve for the state estimate problem and next for the optimal gain. In this paper we express LQG in the $\mathscr{H}_{2}$ framework using the generalised plant configuration, see Fig.6.

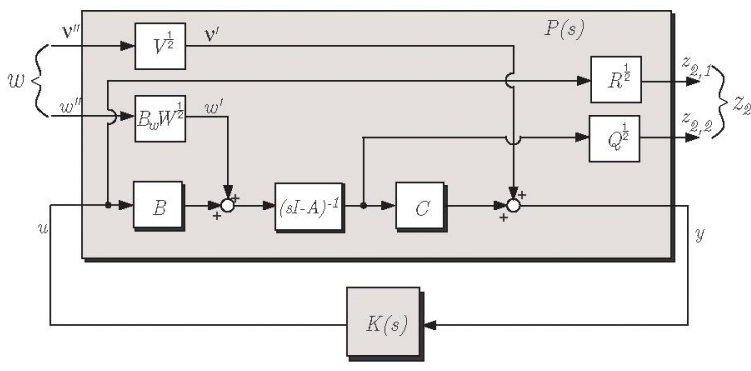

Fig. 6. LQG problem in the generalised regulator configuration

The closed loop $\mathscr{F}_{L}(P, K)$ of the regulated $z_{2}$ (control $z_{2,1}$ and states $z_{2,2}$ ) outputs, to the exogenous inputs (process noise $w^{\prime \prime}$ and sensor noise $v^{\prime \prime}$ ) is given by

$$
\left[\begin{array}{rr}
R^{1 / 2} K(I-G K)^{-1} G_{1} W^{1 / 2} & R^{1 / 2} K(I-G K)^{-1} V^{1 / 2} \\
\left(G_{3}+G_{2} K(I-G K)^{-1} G_{1}\right) W^{1 / 2} & G_{2} K(I-G K)^{-1} V^{1 / 2}
\end{array}\right]
$$

where

$$
\begin{aligned}
G & =C(s I-A)^{-1} B & G_{1} & =C(s I-A)^{-1} B_{w} \\
G_{2} & =Q^{1 / 2}(s I-A)^{-1} B & G_{3} & =Q^{1 / 2}(s I-A)^{-1} B_{w}
\end{aligned}
$$

Then the generalised plant $P(s)$ is given by

$$
P(s) \stackrel{s}{=}\left[\begin{array}{l||ll|l}
A & B_{w} W^{1 / 2} & 0 & B \\
\hline 0 & 0 & 0 & R^{1 / 2} \\
Q^{1 / 2} & 0 & 0 & 0 \\
\hline C & 0 & V^{1 / 2} & 0
\end{array}\right]
$$

The choice of weights usually depends upon the individual application, the experience of the designer and trial-anderror, although references exist on possible systematic ways of choosing the weights and their limitation, for example see [22]. Moreover, the robustness of LQG can be improved by Loop Transfer Recovery (LTR) (the reader is referred to the literature for further details). However, for the purposes of this paper we will follow a simple LQG control exercise.

The choice of the parameters for the current design is: $Q=$ $C^{T} C, R=1, B_{w}=B$ (process noise via the controlled input),
$W=1, V=I$ (three measurements are used). In fact, this usually is a set of simple initial choices found in the context of LQG. The resulting controller is the same size as the reduced plant (recall that we used static weights). The closed loop damping for: (i) the uncontrolled full $\left(138^{\text {th }}\right)$ model, (ii) the designed $\left(9^{\text {th }}\right)$ order controller with the reduced $\left(9^{\text {th }}\right)$ order model, and (iii) the designed controller with the full order model system, are shown in Table I. Although some damping ratios have improved, i.e. for mode 1 and mode 3 , the requirements are not met. In addition, there is a poorly damped mode introduced by the controller in case (iii) at a frequency of $0.470 \mathrm{~Hz}$. Likewise, faster designs will not meet

\begin{tabular}{|c|c|c|c|c|c|c|}
\hline & \multicolumn{2}{|c|}{$\begin{array}{l}\text { Full model } \\
\text { no control }\end{array}$} & \multicolumn{2}{|c|}{$\begin{array}{l}\text { Reduced model } \\
\text { with control }\end{array}$} & \multicolumn{2}{|c|}{$\begin{array}{l}\text { Full model } \\
\text { with control }\end{array}$} \\
\hline mode no. & $\zeta$ & $f(\mathrm{~Hz})$ & $\zeta$ & $f(\mathrm{~Hz})$ & $\zeta$ & $f(\mathrm{~Hz})$ \\
\hline 1 & 0.051 & 0.370 & 0.268 & 0.290 & 0.270 & 0.320 \\
\hline 2 & 0.042 & 0.510 & 0.081 & 0.460 & 0.070 & 0.450 \\
\hline (*) & & - & - & - & $(0.090)$ & $(0.470)$ \\
\hline 3 & 0.047 & 0.590 & 0.100 & 0.556 & 0.120 & 0.550 \\
\hline 4 & 0.050 & 0.790 & & - & 0.050 & 0.790 \\
\hline
\end{tabular}
the requirements (with both damping and robustness issues being critical).

TABLE I

ClOSED-LOOP DAMPING RATIOS $\zeta$ FOR $\mathscr{H}_{2} / L Q G$ (POST-FAULT)

The weights can be manipulated in order to control the speed of response (however we will keep the values fixed for illustration purposes in the design steps). Moreover, to apply Loop Transfer Recovery (LTR) the plant should first be squared by including dummy inputs (details on such a systematic design can be seen in [24]). In such case the design can be substantially improved but the overall procedure can be time-consuming, with designer experience being a key issue.

\section{2 $\mathscr{H}_{2}$ / LQG solution with pole location con- straints via LMIs}

This section extends the previous case of $\mathscr{H}_{2} / \mathrm{LQG}$ control to include closed loop location constraints via LMIs. The weights from the previous design case remain fixed and no additional (dynamic) weights are imposed in the LMI procedure. Thus, the resultant controller is the same order as the design model ( $9^{t h}$ order in this case). The controller was then further reduced to $7^{\text {th }}$ order, using the reduction method discussed in Section 4, with clearly indistinguishable differences from the original $9^{\text {th }}$ order (Fig. 7) (showing by definition the largest singular of the one output/three input controller transfer function). Note that by choosing simple static weights low complexity is maintained and the effectiveness of using LMIs in enhancing the problem formulation is directly illustrated.

Table II lists the closed loop damping ratios for both preand post-fault operating conditions using the reduced $7^{\text {th }}$ order controller (this was actually designed using the reduced post-fault model). The last four rows of the table represent, modes 1,2,3 and 4 respectively. The pole location constraint 


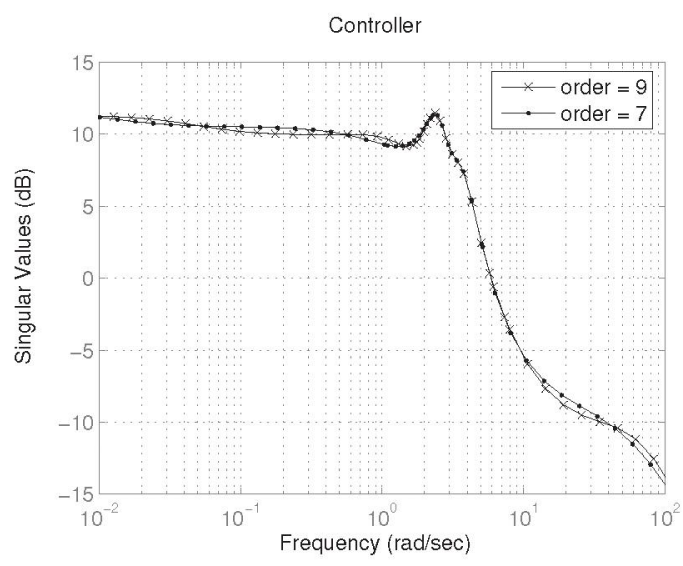

Fig. 7. Designed controller singular value plot

in this configuration was set to at least a damping ratio of $0.1, \theta=2 \cos ^{-1}(0.1)$, (for the design using the reduced order model). It can be clearly seen that although the results have substantially improved from the original $\mathscr{H}_{2} / \mathrm{LQG}$ design, the damping of mode 2 for the pre-fault model marginally fails to meet the requirement of 0.1 .

TABLE II

CLOSED-LOOP DAMPING RATIOS (POLE LOCATION $\zeta$ CONSTRAINT $=0.1$ ) FULL ORDER MODELS WITH REDUCED ORDER CONTROLLER

\begin{tabular}{|c|c|c|c||c|c|c|c|}
\hline \multicolumn{4}{|c||}{ Pre-fault (full) } & \multicolumn{4}{|c|}{ Post-fault (full) } \\
\hline no control & \multicolumn{1}{|c|}{ with control } & \multicolumn{2}{|c|}{ no control } & \multicolumn{2}{|c|}{ with control } \\
\hline$\zeta$ & $f(\mathrm{~Hz})$ & $\zeta$ & $f(\mathrm{~Hz})$ & $\zeta$ & $f(\mathrm{~Hz})$ & $\zeta$ & $f(\mathrm{~Hz})$ \\
\hline 0.063 & 0.391 & $\mathbf{0 . 1 4 0}$ & 0.420 & 0.051 & 0.370 & $\mathbf{0 . 1 2 0}$ & 0.420 \\
\hline 0.044 & 0.508 & $\mathbf{0 . 0 9 5}$ & 0.470 & 0.042 & 0.507 & $\mathbf{0 . 1 3 0}$ & 0.470 \\
\hline 0.055 & 0.623 & $\mathbf{0 . 1 3 0}$ & 0.612 & 0.047 & 0.594 & $\mathbf{0 . 2 4 0}$ & 0.600 \\
\hline 0.050 & 0.792 & 0.055 & 0.790 & 0.050 & 0.791 & 0.055 & 0.785 \\
\hline
\end{tabular}

The next step is to allow for more tolerance when merging the reduced order controller to the full order system structure. Thus, setting the pole location constraint to a minimum damping of $0.2, \theta=2 \cos ^{-1}(0.2)$, (for the design on the reduced order model) satisfies the damping requirements for all modes of interest (i.e. mode 1,2 and 3) as seen from Table III. The assessment is based on the full order models with the reduced order controller.

TABLE III

Closed-Loop damping Ratios (Pole Location $\zeta$ CONSTRAint $=0.2$ ), FULL ORDER MODELS WITH REDUCED ORDER CONTROLLER

\begin{tabular}{|c|c|c|c||c|c|c|c|}
\hline \multicolumn{4}{|c||}{ Pre-fault (full) } & \multicolumn{4}{|c|}{ Post-fault (full) } \\
\hline no control & \multicolumn{2}{|c||}{ with control } & \multicolumn{2}{|c|}{ no control } & \multicolumn{2}{|c|}{ with control } \\
\hline$\zeta$ & $f(\mathrm{~Hz})$ & $\zeta$ & $f(\mathrm{~Hz})$ & $\zeta$ & $f(\mathrm{~Hz})$ & $\zeta$ & $f(\mathrm{~Hz})$ \\
\hline 0.063 & 0.391 & $\mathbf{0 . 1 9 7}$ & 0.380 & 0.051 & 0.370 & $\mathbf{0 . 1 5 4}$ & 0.370 \\
\hline 0.044 & 0.508 & $\mathbf{0 . 1 3 6}$ & 0.470 & 0.042 & 0.507 & $\mathbf{0 . 1 9 5}$ & 0.480 \\
\hline 0.055 & 0.623 & $\mathbf{0 . 1 7 2}$ & 0.610 & 0.047 & 0.594 & $\mathbf{0 . 1 4 5}$ & 0.550 \\
\hline 0.050 & 0.792 & 0.050 & 0.770 & 0.050 & 0.791 & 0.050 & 0.770 \\
\hline
\end{tabular}

Although it seems that by keep increasing the minimum damping constraint results might further improved, in reality this will impose strict constraints on the solution using LMIs for the current application of damping improvement. Usually it will give infeasible solutions or unstable controllers which still stabilise the closed loop (i.e. stabilizing controllers) but clearly impractical to use. For the current application a minimum damping ratio greater or equal to 0.35 initiates this problem). Thus, care should be taken in formulating a reasonable problem setup.

The incorporation of pole location constraints using LMIs for the $\mathscr{H}_{2} / \mathrm{LQG}$ problem, satisfied all damping requirements and also shown good robustness properties as seen from the results. The complexity of the solution was kept low by choosing static weights, in fact the original LQG weights which illustrated the advantages of using LMIs. The design could be seen as a good basis for further robustification, but with the possibility of introducing additional complexity. For example a multi-objective approach in terms of different norms, by incorporating additional constraints for the $\mathscr{H}_{\infty}$ norm of a selected closed loop function (i.e. additional exogenous inputs considered in relation with the existing or new regulated outputs). Emphasis could be placed on selecting possible dynamic weights for the design or other pole location regions (disks, horizontal strips, or combinations of regions etc.). It should be noted that in the case of extra dynamic weights the order of the resultant controller will increase.

\section{Simulation Results}

A first set of results showing the effectiveness of the design has been presented in the previous section. However, this section presents further tests using different operating conditions and a set of time domain results on a non-linear simulation. The controller is the reduced $7^{\text {th }}$ order designed in Section 6 with the pole location constraints (minimum damping constraint $=0.2$ for the reduced order design system). Table IV presents the eigen-analysis results for the closedloop for different line outages scenaria. It is clearly seen that the controller maintains good robustness properties under all probable conditions, despite being designed on a reduced order (nominal) post-fault model. The effectiveness of the LMI solution is clearly evident. (A more detailed robustness assessment in the frequency domain will be presented in the final paper.)

TABLE IV

DAMPING RATIOS FOR A NUMBER OF TIE-LINES OUTAGES WITH 700 MW FLOW BETWEEN NETS AND NYPS AND CI LOAD MODEL

\begin{tabular}{|c||c|c||c|c||c|c|}
\hline \multirow{2}{*}{$\begin{array}{c}\text { Tie-line out- } \\
\text {-age between }\end{array}$} & \multicolumn{2}{c||}{ Mode 1 } & \multicolumn{2}{c||}{ Mode 2 } & \multicolumn{2}{c|}{ Mode 3 } \\
\cline { 2 - 7 } & $\zeta$ & $f(\mathrm{~Hz})$ & $\zeta$ & $f(\mathrm{~Hz})$ & $\zeta$ & $f(\mathrm{~Hz})$ \\
\hline \hline $\mathbf{6 0 - 6 1}$ & 0.144 & 0.380 & 0.180 & 0.480 & 0.159 & 0.540 \\
\hline $\mathbf{5 3 - 5 4}$ & 0.154 & 0.370 & 0.195 & 0.480 & 0.145 & 0.550 \\
\hline $\mathbf{2 7 - 5 3}$ & 0.161 & 0.402 & 0.234 & 0.475 & 0.121 & 0.565 \\
\hline
\end{tabular}

A nonlinear Matlab simulation was carried out over a time-period of $25 \mathrm{sec}$ to demonstrate robust performance of the controller in the presence of nonlinearities inclusive of possible saturation for completeness. Inter-area oscillations are originated due to a three-phase solid fault close to bus \#53 on one of the tie-lines connecting buses \#53-\#54. 
Fig. 8 shows the system dynamic response in terms of the relative angular separation of machine G1 relative to G15, while Fig. 9 the same for machine G15 relative to G13. The system "no control" denotes the absence of supplementary damping, while the primary control loop for the TCSC device continues to sustain the steady-state power-flow specifications. It is illustrated that the inter-area oscillations are successfully damped-out soon after $10 \mathrm{sec}$ with control enabled (10-15sec being the allowed range). Fig. 10 presents the TCSC controller response. Initially the response is quite oscillatory (however within the allowable limits for a TCSC device) due to the nature of the severe line-outage, while settles quickly just after $10 \mathrm{sec}$.

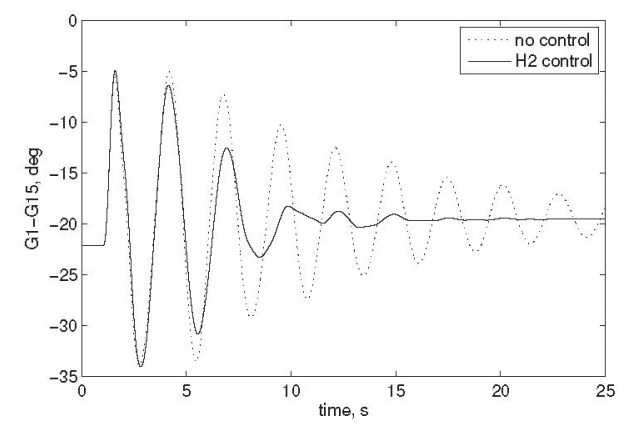

Fig. 8. Machine angle of G1 relative to G15

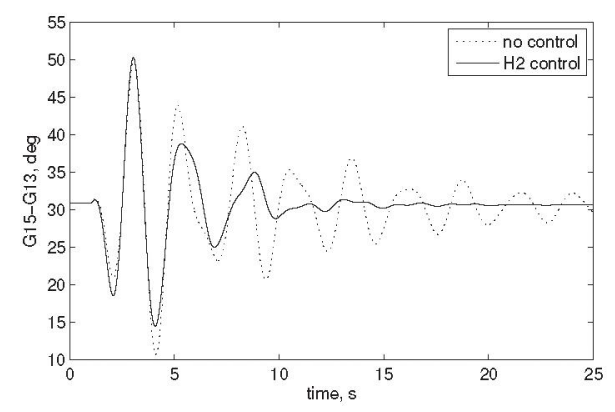

Fig. 9. Machine angle of G15 relative to G13

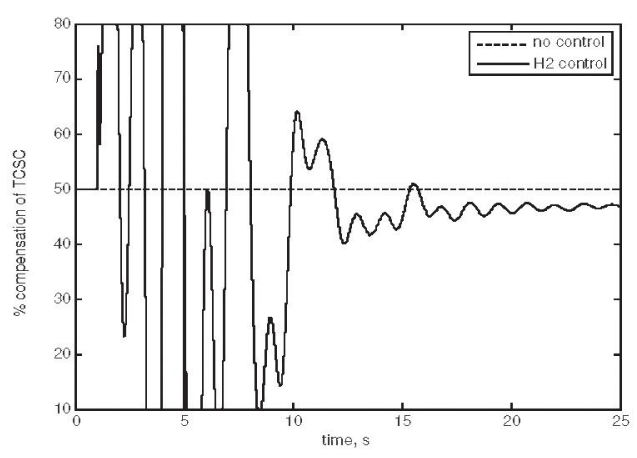

Fig. 10. TCSC controller response

\section{Conclusion}

The paper presented a $\mathscr{H}_{2}$ LMI-based design for improving the damping of power system inter-area oscillations of an equivalent large power system (138th order) using a practical controller structure (7th order) via a TCSC FACTS device. An extensive discussion on effective model reduction for the power system model was presented. The design procedure followed a simple LQG formulation (with the aim to avoid additional complexity) within the $\mathscr{H}_{2}$ framework, and its extension via LMIs incorporating closed loop pole location constraints. The choice of simple static weights (i.e. the ones encountered in LQG-type designs), illustrated the merits of using LMI techniques to impose multiple objectives. Our final design both provided appropriate damping and sustained good robustness properties over the required frequency range and despite the variety of uncertainty/operating conditions in the full non-linear power system model. All required oscillatory modes of interest where sufficiently damped over the range of operating conditions, demonstrated via eigenvalue analysis and time domain simulation results. The design can serve as a useful basis for further extension, i.e. minimisation of $\mathscr{H}_{\infty}$ criteria or additionally providing further robustification (usually at the expense of adding complexity via the extra weighting functions). The paper should be of considerable interest to power system engineers who have to provide practical solutions but may be put off by the potential complexity of advanced control techniques especially in the area of LMIs.

\section{Acknowledgment}

The authors would like to thank Dr Bikash Pal for helpful discussions. Part of this work was supported by the UK Engineering and Physical Sciences Research Council (EPSRC) and by Asea Brown Boveri (ABB) Ltd.

\section{References}

[1] G. Rogers, Power System Oscillations, Kluwer's, 2000.

[2] P. Korba, M. Larsson, A. Oudalov and O. Preiss, Towards the Future of Power System Control, ABB Review Journal, ISSN 1013-3119, no.2/05, pp. 35-38, 2005.

[3] P. Korba, M. Larsson and C. Rehtanz, Detection of Oscillations in Power Systems using Kalman Filtering Techniques, In Proc. of the IEEE Conference on Control Applications, vol. 1, pp. 183-188, Istanbul, Turkey, June 2003.

[4] P. Kundur, Power System Stability and Control, McGraw-Hill, 1993.

[5] H. Werner, P. Korba and T.S. Yang, Robust Tuning of Power System Stabilizers Using LMI-Techniques, IEEE Trans. on Control Systems Technology, vol. 11, no. 1, pp. 147-152, 2003.

[6] R. Sadikovic, P. Korba and G. Andersson, Application of FACTS Devices for Damping of Power System Oscillations, In Proc. of the IEEE PowerTech 2005, St. Petersburg,Russia, June, 2005.

[7] R. Sadikovic, P. Korba and G. Andersson, Self-tuning Controller for Damping of Power System Oscillations using FACTS, In Proc. of the IEEE PES General Meeting, Montral, Canada, June, 2006.

[8] B. Chaudhuri, P. Korba and B.C. Pal, Design of Damping Controllers through Simultaneous Stabilization Technique, Intelligent Automation and Soft Computing (AUTOSOFT), Special Issue on Intelligent Applications in Power Systems, January, 2006. 
[9] M. Farsangi, Y. Song, W. Fang, and X. Wang, Robust FACTS control design using H-infinity loop-shaping method, IEE Proceedings on Generation Transmission and Distribution, Vol. 149 , No. 3, pp. $352-$ $357,2002$.

[10] M. Klein, L. Le, G. Rogers, S. Farrokpay, and N. Balu, H-infinity damping controller design in large power system, IEEE Transactions on Power Systems, Vol. 10, No. 1, pp 158-166, 1995.

[11] B. Pal, A. Coonick, and B. Cory, Robust damping of interarea oscillations in power systems with superconducting magnetic energy storage devices, WEE Proceedings on Generation Transmission and Distribution, Vol. 146, No. 6, pp. 133-639, November, 1999.

[12] B.C. Pal, B. Chaudhuri, A. C. Zolotas, I. M. Jamoukha, and T. Green, Mixed-Sensitivity Approach to H-infinity Control of Power System Oscillations Employing Multiple FACTS Devices, IEEE Transactions on Power Systens, Vol. 18, No. 3,pp 1149-1156, August, 2003.

[13] B.C. Pal, B. Chaudhuri, A. C. Zolotas and I. M. Jaimoukha, Simultaneous Stabilisation Approach for Power System Damping Control Design through TCPAR Employing Global Signals, IEE Proceedings: Generation, Transmission and Distribution, Vol. 151, No. 1, January .pp 43-50, January, 2004.

[14] F. Fatehi, J.R. Smith, and D.A. Pierre, Robust power system controller design based on measured models, IEEE Trans. on Power Systems, Vol. 11, No. 2, pp. 774-780, 1996.

[15] J.R. Smith, F. Fatehi, and D.A. Pierre, Closed-loop identification and tuning for damping of interarea modes, Proceedings of the $33 \mathrm{rd}$ IEEE Conference on Decision and Control, pp. 4055-4060, 1994.

[16] G. Radman, Design of power system stabilizer based on LQG/LTR formulations, Indtistry Applications Sociery Ammal Meeting/Conference Recond of the 1992 IEEE, vol. 2. 1992.

[17] K. M. Son, and J. K. Park, On the Robust LQG Control of TCSC for Damping Power System Oscillations, IEEE Trans. on Power Systems, vol. 15, pp. 1306-1312, 2000

[18] K. Zhou and J. C. Doyle, Essentials of Robust Control, Prentice-Hall, 1998.

[19] J. Paserba, Analysis and control of power system oscillation, CIGRE Special Publication 38.01.07, Vol. Technical Brochure 111, 1996.

[20] I.M. Jaimoukha and E. M. Kasenally, Implicitly Restarted Krylov Subspace Methods for Stable Partial Realizations, SIAM J MATRIX ANAL A, 18 (3),pp. 633-652, July 1997.

[21] S. Boyd, L. El-Ghaoui, E. Feron, and V. Balakrishnan, Linear Matrix Inequalities in System and Control Theory, SIAM, 1994.

[22] B.D.O. Anderson, and J.B. Moore, Optimal Control: Linear Quadratic Methods, Prentice-Hall, 1989.

[23] B. Chaudhuri and B.C. Pal, Robust damping of multiple swing modes employing global stabilizing signals with a TCSC, IEEE Transactions on Power Systems, Vol. 19, No. 1, pp. 499-506, 2004.

[24] A.C. Zololas, B. Chaudhuri, I.M. Jaimoukha, and P. Korba, A study on LQG/LTR control for damping inter-area oscillations in power systems, IEEE Transactions on Control System Technology, 2005 (submitted for publication).

[25] M.G. Safonov, and R.Y. Chiang, A Schur Method for Balanced Model Reduction, IEEE Trans. on Automat. Contr, Vol. AC-34, No. 7, pp. $729-733,1989$.

[26] S. Skogestad and I. Postlethwaite, Mulivariable Feedback Control: Analysis and Desgn, Wiley, 2000.

[27] J.M. Maciejowski, Multivariable Feedback Design, Addison-Wesley, 1989.

[28] P. Gahinet, A. Nemirovski, A. J. Laub, and M. Chilali, LMI Control Toolbor for Use with MATLAB, Mathworks, 1995. 